\title{
BESSRC-CAT Bending Magnet Beamline at the Advanced Photon Source
}

Mark A. Beno", Mark Engbretsona, Guy Jennings", Gordon S. Knapp", Jennifer Linton", Charles Kurtza , Uta Ruett ${ }^{a}$, and Pedro A. Montano $0^{a, b}$

${ }^{2}$ Materials Science Division and Basic Energy Sciences Synchrotron Radiation Center, Argonne National Laboratory, Argonne, IL 60439, USA

bepartment of Physics, University of Illinois at Chicago, Chicago, IL, USA

\section{Materials Science Division \\ Argonne National Laboratory \\ Argonne, IL 60439}

The submitted manuscript has been created by
the University of Chicago as Operator of Argonne
National Laboratony ("Argonne") under Contract
No. W-31-109-ENG-38 with the U.S. Department
of Energy. The U.S. Government retains for
itself, and others acting on its behalf, a peid-up,
non exclusive, irrevocable worldwide license in
said article to reproduce, prepare derivative
works, distribute copies to the public, and
perform publicty and display publicly, by or on
behalf of the Government.

September 2000

jjc

Distribution:

1-2. PRS, 203

3. J. M. Gibson

4. P. A. Montano

5. F. Y. Fradin

6. Office of Science

7. J. Coble

8. Authors

Submitted for publication in Nuclear Instrument and Methods, Section A, from the $7^{\text {th }}$ International Synchrotron Radiation Instrumentation conference (SRI 2000) held August 20-25 in Berlin, Germany.

This work is supported by the US Department of Energy, Office of Basic Energy Sciences, under contract No. W-31-109-ENG-38. 


\section{DISCLAIMER}

This report was prepared as an account of work sponsored by an agency of the United States Government. Neither the United States Government nor any agency thereof, nor any of their employees, make any warranty, express or implied, or assumes any legal liability or responsibility for the accuracy, completeness, or usefulness of any information, apparatus, product, or process disclosed, or represents that its use would not infringe privately owned rights. Reference herein to any specific commercial product, process, or service by trade name, trademark, manufacturer, or otherwise does not necessarily constitute or imply its endorsement, recommendation, or favoring by the United States Government or any agency thereof. The views and opinions of authors expressed herein do not necessarily state or reflect those of the United States Government or any agency thereof. 


\section{DISCLAIMER}

Portions of this document may be illegible in electronic image products. Images are produced from the best available original document. 


\title{
BESSRC-CAT Bending Magnet Beamline at the Advanced Photon Source
}

\author{
Mark A. Beno", Mark Engbretson a , Guy Jennings", Gordon S. Knappa , Jennifer Linton", \\ Charles Kurtz ${ }^{2}$, Uta Rütt and Pedro A. Montano ${ }^{2, b}$,
}

${ }^{a}$ Materials Science Division and Basic Energy Sciences Synchrotron Radiation Center, Argonne National Laboratory, Argonne, II, 60439, USA

bepartment of Physics, University of Illinois at Chicago, Chicago, II, USA

Keywords: Synchrotron Radiation, Spectroscopy, Monochromator

PACS.07.85.Qe, PACS.41. 50.th

PeOPMED

OCT 062000

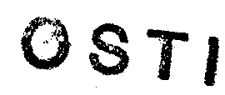

Corresponding Author: Mark A. Beno, Bldg. 433, Argonne National Laboratory

9700 S. Cass Ave, Argonne, IL, 60439, USA

Phone: (630)252-3507, Fax: (630)252-0365, Email: beno@anl.gov

Abstract

The Basic Energy Sciences Synchrotron Radiation Center Collaborative Access Team (BESSRC-CAT) has built a bending magnet beamline for spectroscopy and scattering experiments at the Advanced Photon Source (APS). The windowless beamline uses a water cooled, double crystal, fixed exit monochromator with Si 111 crystals as the first optical element. The monochromator is capable of operations from $55^{\circ}$ to $3^{\circ}$ allowing experiments from approximately 2.4 to $30 \mathrm{keV}$. A monochromatc double mirror system located in the white-beam enclosure focuses the beam in the experimental station. When the first mirror removed energies above the mirror cutoff $(\sim 22 \mathrm{keV})$ can be used. The 12BM-B user station is equipped for scattering (Huber 6-circle diffractometer) and spectroscopy (optical table, ion chambers and solid state detectors) experiments. 


\section{Introduction}

The Basic Energy Sciences Synchrotron Radiation Center is a collaboration of the Argonne National Laboratories Materials Science and Chemistry Divisions along with the Argonne Geoscience group and scientists from the Physics Division of Northern Illinois University. BESSRC has been developed and instrumented to serve the research needs of these users with particular interests in Materials Science, Chemical Science, Atomic Physics and Geosciences. BESSRC has built two sectors with a total of three beamlines at the Advanced Photon Source (APS). One of these is a bending magnet beamline (12BM) which is designed for general spectroscopic, diffraction and scattering experiments.

\section{Beamline Design}

The 12BM beamline has a first optics enclosure (FOE) and a single experimental station at approximately $50 \mathrm{~m}$ from the source. The FOE contains all the major beamline components. The 12BM beamline is window-less so that the beamline vacuum is directly connected to the ring vacuum, allowing the beamline to operate at energies as low as 2.5 $\mathrm{keV}$. The first component inside the FOE is a differential pump which contains a mask limiting the beam to $2.5 \mathrm{mrad}$ horizontal and a vertical aperture of $200 \mu \mathrm{rad}$. The beam size can be further restricted by independent horizontal and vertical white-beam slits which can be used to determine the vertical beam divergence and thus the energy resolution of the monochromator as well as the horizontal beam width which is incident on the monochromator's first crystal. The last white-light component in the FOE is a water-cooled fixed exit double crystal monochromator. The beamline can be operated in 
two modes: either the unfocussed monochromatic beam can be used, or a double mirror system consisting of a flat mirror deflecting the monochromatic beam downward to a toroidal $\mathrm{Rh}$ coated glass mirror can focus the beam into the 12BM experimental station. The last component in the FOE is a white-light beam shutter/ stop. The shielded UHV beam transport ends with monochromatic slits which are located in a small shielded enclosure immediately in front of the 12BM experimental station. The large experimental station ( $6 \mathrm{~m}$ by $3 \mathrm{~m}$ ) has an optiçal table $(1.2 \mathrm{~m} \times 1.8 \mathrm{~m}$ ) for spectroscopic experiments and a Huber 6-circle vertical goniometer for diffraction and scattering investigations permanently installed.

\section{Bending Magnet Monochromator}

The BESSRC 12BM monochromator is a double crystal, fixed exit monochromator with a constant $35 \mathrm{~mm}$ offset designed for UHV operation[1], thereby allowing windowless operation of the beamline. A mechanical linkage and a stepper-motor driven slide are used to correctly position the second crystal and maintain a constant offset. The crystals are mounted on a turntable with the first crystal at the center of rotation. The main drive for the rotary motion is provided by a vacuum compatible Huber 430 goniometer which is isolated from the main vacuum chamber. Rotary motion of the primary monochromator stage is accomplished by using two adjacent vacuum chambers linked only by the small annular opening around a hollow stainless steel shaft which connects the Huber goniometer to the turntable on which the crystals are mounted. This design allows high vacuum operation of the monochromator since it is possible to maintain $10^{-9}$ 
Torr on the monochromator side while maintaining only $10^{-7}$ Torr in the goniometer vacuum chamber. The design consists of two matching perfect crystals mechanically linked by way of two slides mounted orthogonal to each other (a Cowan- Golovchenko linkage[2,3]). The vertical slide for the second crystal mount is driven by a roller at the apex while an in-vacuum stepper motor drives the horizontal slide. In-vacuum stepper motors driven micrometers are used for the $\chi$ and $\theta$ rotations of a kinematic mount on which the second crystal is mounted. An electrostrictive translator mounted in opposition to the theta micrometer provides nearly backlash free fine adjustment of the second crystal for feedback. Large changes in energy tuning are achieved by using the rotary drive mechanism in conjunction with the translation of the second crystal. To scan energy, for example for a spectroscopy experiment, the second crystal drive mechanism is held fixed and the beam diffracted from the first crystal is allowed to walk on the surface of the second crystal. Since the vertical slide of the Cowan-Golovchenko linkage is driven by a mechanical linkage this mode of operation maintains the fixed-exit operation of the monochromator. Water flow cools a copper block onto which the first crystal is clamped. A Ga-In eutectic mixture provides enhanced conduction between the Sil11 first crystal and the cooled copper plate. The water lines are double shielded so that there are no water to ring vacuum joints in the UHV chamber. Because of the high critical energy of the APS bending magnet beamlines, $19 \mathrm{keV}$, Compton scattering heats the second crystal. To eliminate long term thermal drifts from this heating the second crystal is cooled by $\mathrm{Cu}$ braid connected to the monochromator chamber and a radiator plate designed to increase radiative cooling. Front and side views of the monochromator are given in Figures 1 and 2. The monochromator uses Si 111 crystals and is limited to rotation angles from $3^{\circ}$ to $55^{\circ}$ providing energies from 2.4 to $30 \mathrm{keV}$. By using the $\mathrm{Si}$ 333 (third harmonic) reflection energies of 30 to $90 \mathrm{keV}$ are accessible. 


\section{Double Mirror}

The monochromatic double mirror system is designed to permit use of the focussing mirror in the 12BM experimental station without changing the angle of the $\sim 25 \mathrm{~m}$ long shielded UHV beam transport tubes or translation of the monochromatic slits. The use of the double mirror system allows the rapid switching of the beamline from focused to unfocussed mode by raising the first mirror out of the beam. The first downward deflecting flat mirror is a Zerodur flat $40 \mathrm{~cm}$ long, $5 \mathrm{~cm}$ wide and $25 \mathrm{~mm}$ thick coated with a $20 \mathrm{~mm}$ wide Pd film. The second upward deflecting toroidal mirror is $\mathrm{Rh}$ coated silica and is $60 \mathrm{~cm}$ long, $10 \mathrm{~cm}$ wide and $4 \mathrm{~cm}$ thick with a saggital radius of $8 \mathrm{~cm}$. The toroidal mirror is at approximately $29 \mathrm{~m}$ from the source and is at the 1 to 1 position so the fixed saggital radius determines the incident angle of $2.7 \mathrm{mrad}$. At this angle the mirror cutoff is approximately the Rh $\mathrm{K}$ edge energy of $23 \mathrm{keV}$. Both mirrors are mounted on kinematic tables. Only the toroidal mirror has translations to adjust its angle with respect to the incident beam. A 4-point bender adjusts the meridional radius of the toroidal mirror. The mirrors are capable of providing a focus of approximately $0.5 \times 0.5$ $\mathrm{mm}$ with a photon flux of $8 \times 10^{11}$ photons $/ \mathrm{mm}^{2} \mathrm{sec}$ at $10 \mathrm{keV}$.

\section{Experimental Station}

The 12BM-B experimental station is located at $55 \mathrm{~m}$ from the source point. The station is equipped with a kinematic optical table for spectroscopy and a Huber 6-circle also mounted on a kinematic table. The optical table can be translated to give $400 \mathrm{~mm}$ of clearance for the beam and has the capacity ( $>200 \mathrm{~kg}$ ) to be used for large cryostats or magnets. Ion chambers, single and multi-element solid state (Ge) detectors and 
photomultiplyer detectors are available. Ancillary equipment available includes a DISPLEX system, turbo pump, and He flow Cryostat.

Diffraction or scattering experiments in the 12BM-B station on the Huber 6-circle goniometer or spectroscopy experiments on the stations optical table may be controlled with Windows based software [4], a Macintosh based IGOR interface [ 5] or using SPEC [6] on a LINUX system. For the first two operating systems EPICS is used to control the beamline components, for SPEC a BIT3 card in the 12BM-B stations VME crate controls all the stations motors while the monochromator and other beamline components are controlled with an EPICS interface.

\section{ACKNOWLEDGMENTS}

Work at Argonne National Laboratory is supported by the US Department of Energy (DOE), Office of Basic Energy Sciences, Division of Material Sciences, under contract W-31-109-ENG-38.

\section{REFERENCES}

1. M. Ramanathan, M. A. Beno, G. S. Knapp, G. Jennings, P. L. Cowan, and P. A. Montano, Rev. Sci. Instr., , 66 (1995) 2191.

2. J. A. Golovchenko, R. A. Levesque, and P. L. Cowan, Rev. Sci. Instr. 51 (1981) 509.

3. P. L. Cowan, J. B. Hastings, T. Jach and J. P. Kirkland, Nucl. Instr. and Meth. 208 (1983) 349.

4. M. Engbretson, Rapid Application Development (RAD) Software for Synchrotron Beamline Control, in preparation.

5. G. Jennings, Synchrotron Beamline and Instrument Control with IGOR, in preparation.

6. Certified Scientific Software, Cambridge, MA. 


\section{Figure Captions:}

\section{Figure 1}

Front view of the monochromator system. The vacuum chamber is 24 inches in diameter and has 27 inch OD wire sealed UHV flanges. Separate ion pumps are used for each side of the chamber so that a differential pressure of $10^{-3}$ torr can be obtained. The chamber is mounted on a precision kinematic table.

Figure 2

Drawing showing the side view of the BESSRC bending magnet monochromator. The vertical slide of the right angle linkage connecting the two perfect Si 111 crystals is driven by a roller riding on the invar bar which spans the monochromator chamber while the horizontal slide is driven by a rack and pinion gear. Only manual adjustments are used for the initial alignment of the first crystal, but the second crystal can be driven in both $\theta$ and $\chi$ by in-vacuum stepper motors. 
BESSRC CAT DIFFERENTIALLY PUMPED

igure 1 FIXED EXIT MONOCHROMATOR

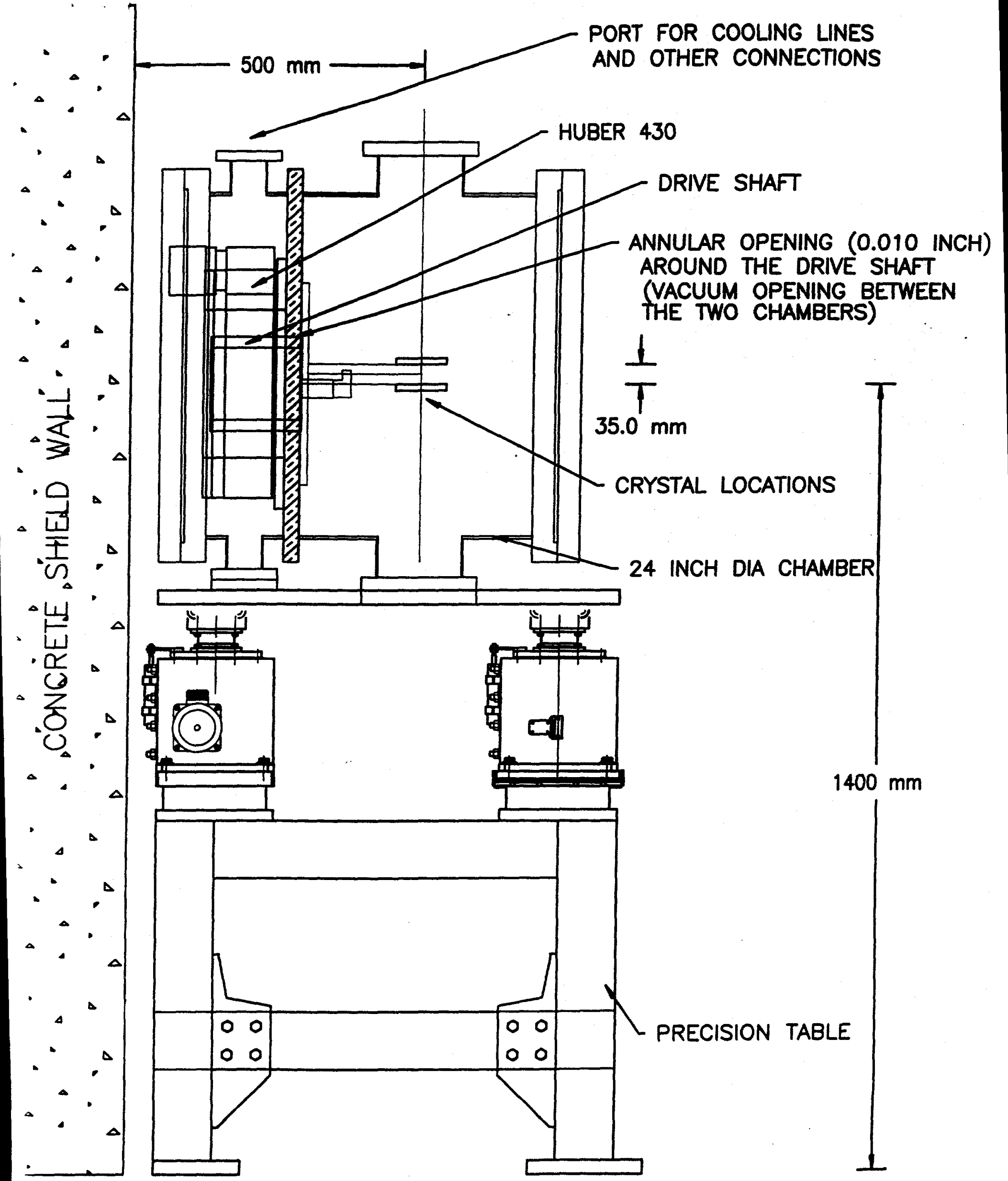




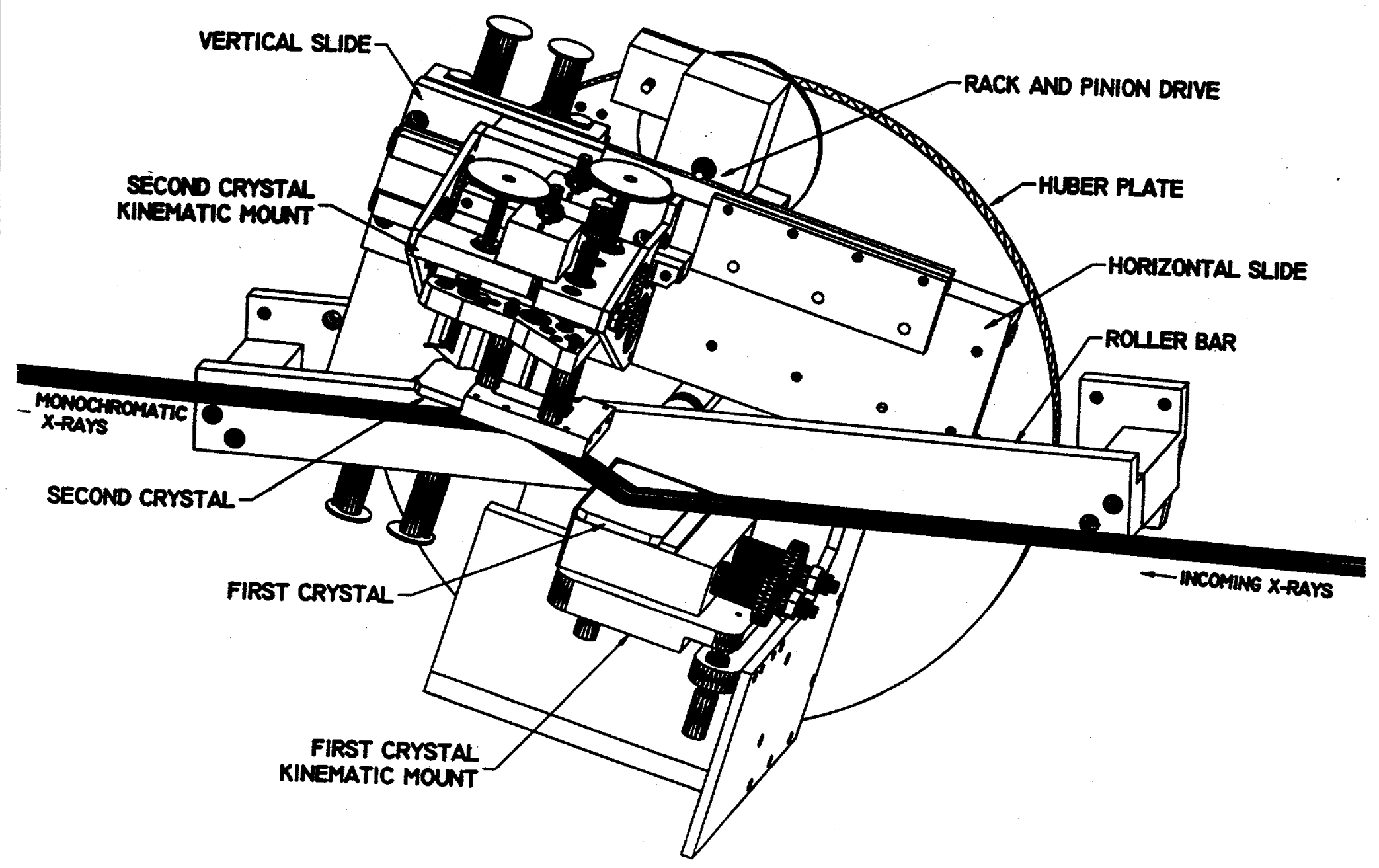

